\title{
Le statut des clitiques sujets cadiens
}

\author{
Francine Alice Girard \\ Université d'Agder - Université d'Oslo \\ francine.girard@uia.no
}

\section{Introduction}

Nous traiterons, dans le présent article, du statut des pronoms sujets clitiques du français cadien, une variété de français parlée dans le sud-ouest de la Louisiane, une région dénommée Acadiana ou encore triangle acadien. Ce triangle, ayant pour base la côte du Golfe du Mexique et pour sommet, la paroisse d'Avoyelles, constitue un espace qui regroupe 22 des 64 paroisses louisianaises depuis la législation de 1968 (Brasseaux 1998 :17). Bien que l'on ne dispose pas de chiffres véritablement fiables (Rousseau 2001 :137), on estime à 250.000 personnes environ le nombre de locuteurs cadiens, descendants pour beaucoup d'Acadiens déportés du Canada en 1755 lors du «Grand dérangement ». Le cadien est un français essentiellement oral, dérivé de dialectes du Grand-Ouest, Poitou et Saintonge principalement, comme le montrent les travaux de Massignon (1962) et Bodin (1987). Les Cadiens ayant vécu quasiment en autarcie jusqu'au début du 20e siècle, leur langue arbore un certain nombre de particularismes intéressants dans une perspective de variation.

Le statut des clitiques sujets dans les langues romanes en général, et en français standard plus particulièrement, a été amplement débattu et depuis Perlmutter (1971), la grammaire générative ne cesse de s'interroger sur la nature des pronoms clitiques. Pour les clitiques du français dit standard ou de référence (FR), les analyses de Kayne (1975, 1983), bien qu'ayant subi un certain nombre de modifications au cours des ans, demeurent aujourd'hui encore les travaux de référence dans le domaine. Kayne propose pour les clitiques sujets du FR une analyse où la cliticisation des proclitiques s'opère en phonologie alors que celle des enclitiques a lieu en syntaxe.

Les recherches portant sur les français parlés informels (FPI) d'où sont absents les enclitiques et où est attesté le redoublement du sujet par un clitique semblent indiquer toutefois que l'analyse de Kayne ne peut s'appliquer à ces variétés. Les travaux de Roberge (1990) portant entre autres sur le FPI québécois et sur le français pied-noir, de Zribi-Hertz (1994) sur le FPI de l'Hexagone, et de Auger (1995) sur le FPI québécois mènent ces chercheurs à argumenter en faveur d'une cliticisation syntaxique/morphosyntaxique, le proclitique sujet ayant le statut d'affixe flexionnel marqueur d'accord.

Nous discuterons ici dans quelle mesure les caractéristiques du système pronominal sujet du cadien font qu'il s'apparente ou non à celui de ces français parlés informels et s'il partage la même grammaire.

\section{Le statut des clitiques sujets en FR}

Dans son analyse détaillée du comportement des pronoms sujets du FR, Kayne (1975) montre que ceux-ci peuvent apparaître en position préverbale et en position postverbale. Les différences de comportement observées entre proclitiques et enclitiques le mènent (Kayne 1975 et 1983) à opérer avec deux analyses distinctes de la cliticisation des pronoms sujets en FR.

\subsection{Le statut des proclitiques}

Kayne accorde aux proclitiques sujets le statut d'argument. Ils sont de véritables sujets, au même titre que les SN sujets. L'hypothèse de Kayne repose essentiellement sur les deux faits suivants :

- la complémentarité des SN et des clitiques sujets, le proclitique nominatif ne pouvant pas être employé en cooccurrence avec le SN sujet comme l'illustre l'exemple (1) :

(1) *Jean il viendra. 
- tout comme le SN sujet, le proclitique peut s'ellipser dans les structures coordonnées :

(2) Jean viendra et présentera son nouveau livre.

(3) Il viendra et présentera son nouveau livre.

Dans l'analyse de Kayne, le proclitique est considéré comme un argument pronominalisé et déplacé de sa position profonde de sujet vers une position adjointe au verbe. Il est cliticisé en phonologie à l'issue d'une règle phonologique tardive, postsyntaxique.

\subsection{Le statut des enclitiques}

Pour ce qui est des enclitiques, Kayne (1983) fait remarquer que leur syntaxe se distingue de celle des proclitiques essentiellement par deux points:

- l'enclitique peut apparaître en cooccurrence avec le SN sujet :

\section{(4) Jean viendra-t-il ?}

- l'enclitique ne peut pas s'ellipser :

(5) Jean viendra-t-il et présentera-t-il son nouveau livre ?

(6) *Jean viendra-t-il et présentera son nouveau livre?

Ces deux caractéristiques des enclitiques nominatifs signalent qu'ils ne sont pas des arguments, mais des clitiques dès la structure profonde, ce qui mène Kayne à argumenter en faveur d'une cliticisation syntaxique de ces enclitiques.

Nous verrons maintenant comment les recherches portant sur les français parlés informels sembleraient indiquer que l'analyse de Kayne ne peut s'appliquer à ces variétés de français.

\section{Le statut des clitiques en FPI}

Pour illustrer la situation des FPI, nous résumerons les travaux de Zribi-Hertz (1994) sur le français parlé informel de l'Hexagone et de Auger (1995) sur le français parlé informel québécois et donnerons un rapide aperçu du français pied-noir décrit par Roberge (1990).

\subsection{Le statut des clitiques dans le français parlé informel de l'Hexagone}

Zribi-Hertz (1994) note que, dans le français parlé informel de l'Hexagone (FPIH), les proclitiques sujets:

- ne sont pas ellipsables dans les structures coordonnées :

(7) Il mangera beaucoup de viande et *boira beaucoup de vin.

Il a mangé beaucoup de viande et **a bu beaucoup de vin.

- peuvent apparaître en cooccurrence avec un sujet lexical, l'absence de pause entre le SN sujet et le clitique indiquant qu'il s'agit d'un redoublement du sujet et non d'une dislocation :

(8) Pierre il mange. (un patron de phrase possible)

- peuvent apparaître en cooccurrence avec un sujet quantifié comme dans les exemples en (9):

(9) Tout le monde il est beau, tout le monde il est gentil.

Personne il m'aime. 
Ce dernier point, nous le verrons, constituera l'élément central de l'argumentation de Zribi-Hertz car c'est là le critère essentiel dans la théorie Principes et Paramètres pour établir la distinction entre dislocation et redoublement du sujet par un clitique.

Pour rendre compte des différences de comportement observées entre les proclitiques du FPIH et du FR, Zribi-Hertz (1994) argumente en faveur de deux grammaires distinctes pour ces deux variétés de français et précise que (Zribi-Hertz 1994 : 137) « tous les travaux récents portant sur la syntaxe du français avancé (FA) indiquent de façon convergente que la structure de la proposition fléchie y est en train de devenir semblable à celle du trentin ».

Dans ce dialecte du nord de l'Italie décrit par Rizzi (1986) et Brandi et Cordin (1989), le SN sujet est obligatoirement doublé d'un clitique ainsi que l'illustrent les deux phrases en (10)a et b :

(10)

\section{a. El Gianni el magna. \\ Le Gianni il mange}

'Gianni mange

\section{b. *El Gianni magna.}

Les proclitiques sujets sont en conséquence analysés non pas comme des arguments mais des épels d'accord ainsi que le montre la structure en arbre donnée en (11) où le clitique est généré sous $\mathrm{AGRs}^{\circ}$ dans la structure de base :

(11)

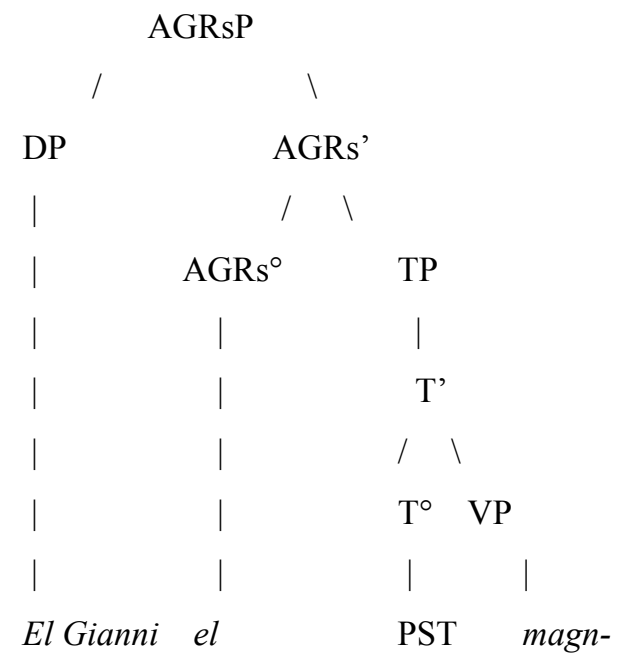

Zribi-Hertz montre que les proclitiques du FA, tout comme les enclitiques du FS décrits par Kayne (1975), sont solidaires de la flexion verbale, ce qui les identifie comme des éléments fonctionnels, des épels de l'accord-sujet et non des sujets. Elle en conclut que le proclitique sujet du FA est engendré directement dans la position $\mathrm{AGRs}^{\circ}$.

Zribi-Herz propose ainsi deux analyses différentes pour le pronom $i l$ de (12a) et celui de (12b).

\section{(12)}

$$
\begin{aligned}
& \text { a. Il mange. (FS moderne) } \\
& \text { b. Il mange. (FA) }
\end{aligned}
$$

Dans (12a), Il ne participe pas de la flexion verbale et est engendré dans la position Spec, AGRP, pour être ensuite cliticisé tardivement au syntagme verbal. Zribi-Hertz suit en cela l'analyse de Kayne (1975). Dans (12b), en revanche, $i l$ est engendré sous $\mathrm{AGRs}^{\circ}$ et doit sa cliticité à son statut flexionnel. 


\subsection{Le statut des clitiques sujets dans le français parlé informel québécois}

La situation du français parlé informel québécois (FPIQ) est en plusieurs points comparable à celle du FPIH. Auger (1995) observe effectivement que les clitiques du FPQI arborent un comportement analogue à ceux d'autres français parlés informels et argumente elle aussi pour leur caractère non-argumental. Auger étaye son hypothèse à l'aide d'arguments similaires à ceux de Zribi-Herz :

- les clitiques sujets du FPQI ne sont pas ellipsables dans les structures coordonnées :

\section{(13) Je mange du pain pis je bois du vin.}

- les clitiques du FPQI s'emploient en cooccurrence avec le SN sujet sans qu'il y ait de pause entre le SN et le clitique, ce qui indique, nous l'avons vu en 3.1., qu'il ne s'agit pas de dislocation mais d'un redoublement du sujet :

\section{Les maringouins ils me suivent.}

\subsection{Le statut des clitiques sujets dans le français pied-noir}

Roberge (1990) argumente pour le statut affixal des clitiques sujet de différentes variétés de français et montre entre autres que le français pied-noir autorise, comme le FPIQ, le redoublement optionnel du sujet par un clitique ainsi que le montrent les exemples ci-dessous :

\section{L'homme (il) vient.}

\section{Le soleil (il) brille pour tout le monde.}

Ce dialecte permet en outre le redoublement des $\mathrm{SN}$ indéfinis et quantifiés comme l'attestent les phrases en (16) :

\section{Un homme il vient.}

Chaque femme elle parle.

Personne il sait qui est leur mère.

\subsection{Sujets quantifiés et le statut des clitiques sujets}

Les données sur lesquelles se fondent les analyses présentées ci-dessus ont toutefois été critiquées entre autres par Côté (2001) dans un article intitulé On the Status of Subject Clitics in Child French. Cette critique, que nous résumerons ici avant d'examiner les données cadiennes et de proposer une analyse pour cette variété de français, porte essentiellement sur l'interprétation des sujets quantifiés dans les articles de Zribi-Hertz (1994) et de Auger (1995).

En effet le statut non-argumental du sujet clitique repose essentiellement sur les faits suivants :

- la présence du clitique sujet est obligatoire devant le verbe

- la présence du clitique sujet est obligatoire dans les structures coordonnées

- le redoublement du sujet.

C'est en fait ce dernier argument qui pèse le plus en faveur des analyses de Zribi-Hertz et de Auger. Le fait qu'un sujet quantifié soit doublé indiquerait qu'il ne s'agit pas d'un cas de dislocation à gauche mais bien de redoublement puisque c'est là le critère essentiel dans la théorie Principes et Paramètres pour établir la distinction entre dislocation et redoublement du sujet par un clitique.

Les sujets quantifiés du français comprennent les sujets indéfinis (des SN introduits par un, quelques, aucun, rien, personne, les chiffres) et les quantifieurs universaux (tout/tous et chaque/chacun). 
Côté (2001) souligne qu'en réalité tous les SN indéfinis ne sont pas exclus des dislocations en FR. En effet, seuls les SN indéfinis à valeur existentielle le sont, les SN indéfinis à valeur générique pouvant être librement disloqués. Ainsi l'exemple (17) est inacceptable en FR alors que (18) est acceptable :

\section{*Certains briquets, ils ont du gaz.}

Une voiture, ça roule.

De même tout/tous peut avoir des emplois de non-quantifieur qui sont autorisés en dislocation en FR. Côté (2001) propose d'analyser tout/tous déterminant comme un quantifieur lorsqu'il n'apparaît pas avec un 'vrai' déterminant défini (l'article possessif et démonstratif et l'article défini en dehors de son emploi générique). Ceci explique selon elle l'acceptabilité de (19) et l'inacceptabilité de (20) :

(19) Toute cette pluie-là, elle me rend malade.

Tous tes enfants, ils y sont allés?

*Tous les hommes, ils sont mortels.

Pour Côté (2001), ces distinctions n'ont pas été prises en compte par Zribi-Hertz (1994) et Auger (1995) dans leur discussion sur le statut des clitiques dans ces parlés informels, ce qui sape le fondement même de leurs analyses.

Le cadre de cet article ne nous permet pas d'examiner plus en détails la pertinence de cette critique pour l'analyse des clitiques du FPIH et du FPIQ comme affixes. Le statut des clitiques sujets du français cadien étant notre objet ici, nous présenterons maintenant les caractéristiques du système pronominal cadien pour ensuite montrer comment l'analyse proposée en (3) peut s'appliquer à cette variété de français, les données du cadien montrant que le comportement de ces clitiques sujets ne tombe pas sous la critique de Côté (2001).

\section{Les clitiques sujets en français cadien}

Le français cadien présentant un grand degré de variation, nous avons limité notre étude à l'une des deux grandes zones francophones cadiennes telles qu'elles sont définies dans les travaux de Brasseaux (1992) et (1998) et Breton (1998). Les données cadiennes présentées dans cette section proviennent de la zone centrée autour de Lafayette, région qui correspond à la première vague d'immigration acadienne, la deuxième grande zone francophone cadienne, les régions côtières des bayous Lafourche et Terrebonne, ayant déjà fait l'objet d'une étude approfondie (Rottet 2001). Nos exemples sont extraits d'un corpus oral que nous avons recueilli en 1997 dans cette région et du corpus de LSU constitué sous la direction de la professeure Dubois. À cela s'ajoutent quelques exemples tirés des contes cadiens transcrits par Ancelet dans Cajun and Creole Folktales (1994), de Cadjins et créoles en Louisiane de Griolet ainsi que de la Louisiana French grammar de Conwell et Juilland (1963).

L'étude de ces données montre que le comportement des clitiques sujets du cadien présente un certain nombre de similarités avec ceux du FR. Tout comme ces derniers, ils ne peuvent être coordonnés ni modifiés, ils ne peuvent s'employer isolément et ils ne peuvent fonctionner comme sujet d'une proposition participe absolue ou d'un infinitif exclamatif. Le système pronominal cadien se distingue toutefois en plusieurs points de celui du FR.

\subsection{Réduction du paradigme sujet clitique}

La première particularité qui s'impose est la réduction considérable du paradigme sujet clitique au profit des pronoms personnels toniques et du pronom ça. 


\subsubsection{Remplacement des clitiques par le pronom tonique}

$\mathrm{Si}$ ce phénomène touche plus particulièrement les clitiques pluriels nous et vous qui sont systématiquement remplacés respectivement par nous-autres et vous-autres comme l'illustrent les phrases en (21):

\section{Nous-autres parlent pas comme ça.}

\section{Vous autres allent pas à des bals ?}

nous pouvons également observer une généralisation de l'emploi de formes sujets non-clitiques à la deuxième personne du singulier et à la troisième personne du singulier et du pluriel :

\section{Quand toi es venu. (Conwell et Juilland 1963) \\ Lui travaille. (Conwell et Juilland 1963) \\ Eux-autres va jamais au movie.}

Seules les formes je et elle semblent persister dans la variété de cadien présentée ici où l'on note des phrases comme :

\section{J'i dis ça.}

Elle avait sept ans plus vieux que moi.

\subsubsection{Extension du champ d'emploi du pronom ça}

Le champ d'emploi du pronom ça, forme non-clitique, connait une expansion considérable en cadien au détriment de celui des clitiques dont il a envahi plusieurs domaines ainsi que l'illustrent les exemples de (24) à (26) :

(24) Elle, ça dit la bonne aventure. (Conwell et Juilland 1963)

Ça fait, ça [mes petits-enfants] parle pas français.

\section{Un Cadien [générique] ça travaillait pas, ça buvait, ca buvait tout le temps.}

Les Cadiens ça fait la boucherie.

Ça mouille.

Ça neigeait.

Dans (24), ça remplace les clitiques de la troisième personne du singulier et du pluriel, même lorsqu'ils réfèrent à des êtres animés définis, phénomène observé déjà par Conwell et Juilland (1963) et Brown (1988).

Dans (25), ça renvoie à une troisième personne du singulier dans le premier exemple et du pluriel dans le second avec, dans les deux cas, un sens indéfini et exclusif. Il fonctionne ici comme un pronom générique.

Dans (26), ça fonctionne comme le pronom impersonnel $i l$ du FR devant les verbes indiquant le temps qu'il fait.

Il faut noter aussi que, à la différence du FR, les emplois de ça illustrés dans cette section ne sont pas soumis à des contraintes d'ordre stylistique. Brown $(1988$ :124) observe en effet : «Based on empirical evidence gathered in the field, there is no reason to believe at this point that it (ça) carries a stylistic marking for any particular speech style.» 


\subsection{Réduction phonétique du clitique sujet}

La réduction phonétique des formes sujets clitiques est systématique en cadien et ceci indépendamment de la nature du segment qui suit le pronom, que ce soit l'élément verbal comme en (27) ou un autre clitique comme en (28):

J'va pas aux bals.

Mom l'[elle] a jeté ses pills.

$\mathbf{N}^{\prime}[0 n]$ a passé beaucoup d'bon temps sur la galerie.

J'i [je lui] ai dit ça.

\subsection{Effacement du clitique sujet}

On trouve en cadien des phrases à sujet nul - tout du moins en structure de surface - par suite de l'effacement du clitique sujet. Cet effacement est toutefois soumis à des restrictions particulières lorsqu'il ne s'agit pas des expressions quasiment lexicalisées que l'on trouve en (29) où l'on emploie y a pour il y $a$ et faut et fallait pour il faut et il fallait :

(29) Y a du monde ils vont pas aller si ils vont pas boire.

$Y$ en a des tout gris.

Pour aller à l'école, fallait je marche.

Dans les autres cas, le clitique sujet effacé doit être recouvrable dans le contexte. L'effacement peut ainsi avoir lieu dans l'exemple (30) en raison de la présence du clitique réfléchi, ce dernier étant porteur des traits de nombre et de personne.

Me souviens. (Conwell et Juilland 1963)

\subsection{Reprise du clitique sujet dans les structures coordonnées}

Le clitique sujet n'est pas effacé dans les structures coordonnées lorsqu'il y a identité comme l'indiquent les phrases en (31) :

(31) Ils chassaient et ils trouvaient pas grand chose à tirer.

Je travaillais en l'été avec mon beau-père et je mettais les briques.

\subsection{Absence d'enclitiques}

On ne trouve en cadien aucune forme d'inversion ni simple ni complexe et les exemples en (32) montrent que l'on retrouve dans les questions le même ordre sujet-verbe que dans les phrases affirmatives :

(32) Mais quoi il faisait ?

Quoi c'est qui est arrivé ici ?

Mais quoi ça fait ?

Le cadien n'inverse pas non plus dans les incises, qu'elles soient placées après ou à l'intérieur du discours direct ainsi qu'en témoignent les phrases en (33) :

(33) « Mom, si je retourne pas back », il dit, « lâche mes chiens.» (Ancelet 1994)

«Ah, j’peux mourir en paix », i(l) dit. (Griolet 1986) 


\title{
4.6 Redoublement du sujet
}

Nous avons relevé dans notre corpus de nombreux cas de redoublement du sujet, les proclitiques sujets apparaissant en cooccurrence avec un SN sujet et l'absence de pause indiquant qu'il ne s'agit pas de dislocation à gauche. Ceci n'est pas seulement le cas des proclitiques de la première personne du singulier, phénomène déjà noté par Dubois (2001) et Rottet (2001) :

(34) Moi j’ai mangé. (Dubois 2001 :159)

Nous avons en effet enregistré dans notre corpus de nombreux exemples de sujet doublé à la deuxième personne du pluriel et à la troisième personne du singulier et du pluriel comme l'illustrent les phrases en (35) :

\author{
Pop il veut pas aller au bal. \\ Mom I' [elle] a jeté ses pills. \\ Nous-autres n’ [on] a dansé sur la galerie. \\ Ce monde ça dépense pas d'argent.
}

Personne il a rien dit.

Un Cadien ça travaillait pas.

Lui et Jebby ils jouaient tout le temps.

Les Cadiens ça fait la boucherie.

Tout quelqu'un [tout le monde] ils faisaient ça.

\section{Conclusion : le statut des clitiques sujets cadiens}

Les particularités du comportement des clitiques cadiens que nous avons décrites en 4. s'apparentent en plusieurs points à celles rapportées pour le FPIH par Zribi-Hertz (1994), par Auger (1995) pour le FPIQ et par Roberge (1990) pour le français pied-noir.

Nous avons pu constater en cadien :

- l'absence d'enclitiques

- la présence obligatoire du clitique sujet dans les structures coordonnées

- le redoublement du sujet

C'est sur ce dernier point, crucial pour l'analyse comme nous l'avons vu, que nous nous attarderons. La critique de Côté (2001) portait principalement sur la non-prise en compte des différentes interprétations des SN indéfinis et des quantifieurs, ce qui invalidait entre autres l'analyse de Zribi-Hertz.

La situation est différente pour le cadien où est attestée la dislocation de SN indéfinis à interprétation existentielle comme le montrent les exemples en (36) :

\section{Personne il a rien dit.}

Tout quelqu'un [tout le monde] ils faisaient ça.

Ceci nous permet de conclure que la critique de Côté (2001), qui réfutait pour le français avancé une analyse inspirée de celle des dialectes du nord de l'Italie, ne s'applique pas au cadien. Nous pouvons donc proposer pour les pronoms sujets cadiens une cliticisation non pas phonologique ainsi que Kayne (1975) l'avait fait pour les proclitiques du FR, mais d'ordre syntaxique/morphosyntaxique dont la représentation s'apparentera à celle donnée pour le trentin. En effet, les pronoms personnels sujets du cadien pouvant apparaitre en cooccurrence avec le SN sujet, ils ne peuvent avoir le statut d'argument que Kayne (1975) conférait aux proclitiques du FS et doivent être analysés comme des éléments fonctionnels, des épels d'accord. 


\section{Syntaxe}

DOI $10.1051 / \mathrm{cmlf} / 2010209$

L'exemple (37) aura en conséquence la structure en arbre ci-dessous:

(37)

Moi je mange.

AGRsP

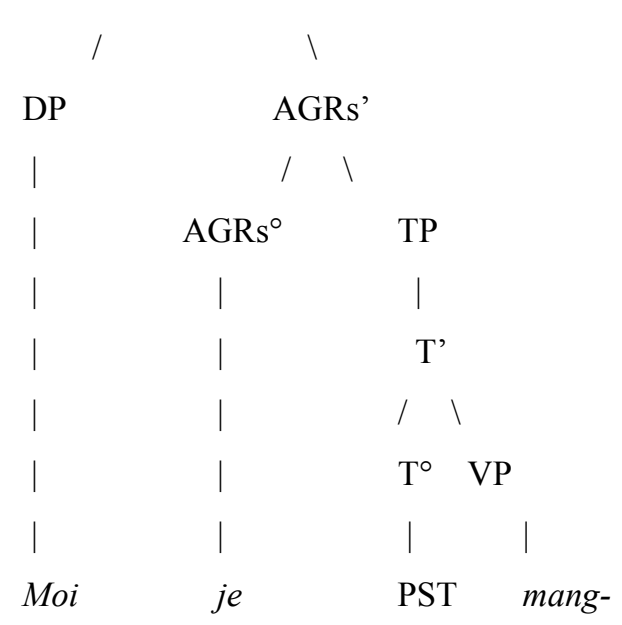

Nous noterons pour conclure que le redoublement du sujet en cadien, s'il est fréquent dans notre corpus, n'est qu'un patron possible et qu'il coexiste avec la structure sans redoublement. Il sera donc intéressant d'étudier dans quelle mesure ce redoublement est productif ou s'il est en perte de terrain.

Rottet (2001) attribue cet emploi à des locuteurs dont la compétence en français est très restreinte. Ceci n'est pas corroboré par l'analyse de nos données où le redoublement du sujet est attesté - à un plus ou moins grand degré - chez tous nos locuteurs indépendamment du sexe, de l'âge et de la compétence en français.

Ce phénomène se retrouvant, nous l'avons vu, dans plusieurs variétés de français parlé informel, s'agirait-il d'un phénomène de français avancé pour reprendre le terme de Frei (1929)?

Côté (2001) signale par ailleurs que les exemples de redoublement du sujet dans les FPI cités par Renzi (1992), Vanelli (1987) et Zribi-Hertz (1994) et repris en (38) proviennent de sources écrites anciennes :

(38) Tous ils veulent venir. (Renzi 1992 ; de Bauche 1920)

Chacun il a sa chimère. (Vanelli 1987 ; de Sanfeld 1928)

Personne il fiche rien à Toulon. (Zribi-Hertz 1994 ; de Mille 1908)

La présence de cette structure en cadien pourrait alors illustrer la persistance d'un trait archaïque.

Une analyse approfondie de ces phénomènes dans notre corpus tentera d'apporter une réponse à ces questions.

\section{Références bibliographiques}

Ancelet, B. (1994). Cajun and Creole Folktales, New York : Garland Publishing.

Auger, J. (1995). Les clitiques pronominaux en français parlé informel: une approche morphologique, Revue québécoise de linguistique, 24, 1, 21-60.

Bodin, C. (1987). The Dialectal Origins of Louisiana Acadian French, UMI (University Microfilms) Dissertation Services.

Brandi, L. et Cordin, P. (1989). Two Italian dialects and the null subject parameter, dans : O. Jaeggli and K. Safir (eds) The Null Subject Parameter. Dordrecht: Kluwer, 111-142. 
Brasseaux, C. (1992). Acadian to Cajun: Transformation of a People, 1803-1877, Jackson : University Press of Mississipi.

Brasseaux, C. (1998). Acadian Settlement Patterns, 1765-1900, dans Creoles and Cajuns: French Louisiana - La Louisiane Française, Frankfurt am Main : Peter Lang.

Breton, Roland J.-L. (1998). Comment mesurer l'extension géographique de la francophonie en Louisiane? dans Creoles and Cajuns: French Louisiana - La Louisiane Française, Frankfurt am Main : Peter Lang.

Brown, B. (1988). Pronominal Equivalence in a Variable Syntax, Unpublished dissertation, University of Texas at Austin.

Conwell, M. et A. Juilland, (1963). Louisiana French Grammar, La Haye : Mouton.

Côté, M.-H. (2001). On the Status of Subject Clitics in Child French, dans M. Algren, A. Barresa, M.-J. Ezeizabarrena, I. Idiazabal and B. MacWhinney (eds) Research on Child Language Acquisition, Sommerville : Cascadilla Press, 1314-1330.

Dubois, S. (2001). Attrition linguistique ou convergence dialectale : JE, MOI/JE et MOI en français cadien, Revue de linguistique, 18, 149-165.

Frei, H. (1929). La grammaire des fautes, Genève : Slatkine.

Griolet, P. (1986). Cadjins et créoles en Louisiane, Paris : Payot.

Kayne, R. (1975). French Syntax : the Transformational Cycle, Cambridge (Mass) : MIT Press.

Kayne, R. (1983). Chaînes, catégories extérieures à $\mathrm{S}$ et inversion complexe en français, Langue française, 58, 36-65.

Massignon, G. (1962). Les parlers français d'Acadie, Paris : Klincksieck.

Perlmutter, D. (1971). Deep and Surface Structure Constraints in Syntax, New York : Holt, Rinehart and Winston.

Rizzi, L. (1986). On the status of subject clitics in Romance, dans : O. Jaeggli et C. Silva Corvalàn (eds) Studies in Romance Linguistics, Dordrecht : Foris, 391-419.

Roberge, Y. (1990). The Syntactic Recoverability of Null Arguments, Kingston : McGill-Queen's University Press.

Rottet, K.J. (2001). Language Shift in the Coastal Marshes of Louisiana, New York : Peter Lang.

Rousseau, A. (2001). Les Cadjins de la Louisiane, destin d'une communauté francophone des États-Unis, une interpretation géographique, Thèse de doctorat, Université de Paris-Sorbonne.

Zribi-Hertz, A. (1994). La syntaxe des clitiques nominatifs en français standard et avancé, Travaux de linguistique et de philologie, 32, 131-147. 